\title{
ENC Measurement for ASIC Preamp Board as a Detector Module for PET System
}

\author{
N. Nagara* \\ Center for Applied Nuclear Science and Technology, National Nuclear Energy Agency \\ Jl. Tamansari No. 71, Bandung 40132, Indonesia
}

\section{ARTICLE INFO}

Article history:

Received 22 October 2014

Received in revised form 23 Februari 2016

Accepted 3 March 2016

Keywords:

Positron emission tomography

Equivalent noise charge

Application-specific integrated circuit

Avalanche photodiodes

\begin{abstract}
A B S T R A C T
We developed a gamma ray detector with an LuAG:Pr scintillator and an avalanche photodiode as a detector for a positron emission tomography (PET) system. Studies have been performed on the influences of gamma irradiation on application-specific integrated circuit (ASIC) preamp boards used as a detector module. As a device used in nuclear environments for substantial durations, the ASIC has to have a lifetime long enough to ensure that there will be a negligible failure rate during this period. These front-end systems must meet the requirements for standard positron emission tomography (PET) systems. Therefore, an equivalent noise charge (ENC) experiment is needed to measure the front-end system's characteristics. This study showed that minimum ENC conditions can be achieved if a shorter shaping time could be applied.
\end{abstract}

\section{INTRODUCTION}

For many years, scintillation detection has been based almost exclusively on the light readout by photomultipliers tube (PMT). The scintillation detection is an important technique in research, medicine, and industry. The high gain, low noise, and fair quantum efficiency of the PMTs allow them to be used with different scintillators. For some selected applications, mainly in high-energy physics, the photodiode has been proposed and successfully applied with in organic scintillators [1].

Compared to photomultiplier tubes, avalanche photodiodes (APD) have some advantages, namely: low temperature sensitivity; pixel miniaturization, as the APD's thickness is less than $1 \mathrm{~mm}$ and its weight is only a few grams; higher optical quantum efficiency by a factor of 3; and ability to function in high magnetic fields. However, APDs have a relatively small sensitive area (several $\mathrm{cm}^{2}$ ) and their gain is moderate $\left(<10^{4}\right)$. Because of the relatively small gain, a charge-sensitive preamplifier (CSA) is needed; however, the CSA smears the very

* Corresponding author.

E-mail address: : nanddaa@batan.go.id

DOI: http://dx.doi.org/10.17146/aij.2016.506 fast rise time $(<2 \mathrm{~ns})$ of the APDs [2]. A gammaray detector with LuAG:Pr scintillator for positron emission tomography (PET) system has been developed by using an APD as a detector (front-end) module [3].

We build small PET system with total 8 detector board, which consist of 48 channels for every board. As an equipment for use in nuclear environments for significant durations, the lifetime of the application-specific integrated circuit (ASIC) has to be long enough to ensure that there will be negligible failure rate during this duration. This front-end system must match the requirements for standard PET system. Therefore, an equivalent noise charge (ENC) experiment is needed to measure the front-end characteristics.

\section{EXPERIMENTAL METHODS}

There were several research activities and practical experiences in front-end system development namely (1) construction of UVenhanced APD array (Hamamatsu S8664 8223) coupled with Pr:LuAG scintillator as the detector module, (2) construction of data processing module of the Time-based Front-end System using ASIC 
technologies, (3) counting digital output signal by using data acquisition module (Cyclone III FPGA by Altera). For data processing module, a 48channel time-based ASIC has been built for this research. The designed ASIC is different from the common time-based front-end electronics which only contains a charge-sensitive preamplifier (CSA) and time-over-threshold (ToT) circuit for each channel. Consisting of a detector module, a data processing module and a data acquisition module, a PET system requires a front-end electronics with low noise and high counting rate. Therefore, the characteristics of the preamplifier significantly influence the figure of merit for the overall electronics.

The noise level of the preamplifier is usually represented by its equivalent noise charge (ENC). Typically expressed in fC, electrons, or eV, ENC is defined as the magnitude of the step function of charge supplied to the CSA's input that will produce an output pulse whose peak amplitude is equal to the root mean square (RMS) noise voltage at the output.

ENC measurements were conducted for the irradiated ASIC preamp board which is part of the module detector. For this measurement, we built an experimental board with a size of $95 \mathrm{~mm} \times 95 \mathrm{~mm}$ consisting of 48 input channels and 48 output channels (Fig. 1). The input pulse was generated by a precision pulse generator with a positive direct output. These pulse signals were converted into charge signals using a $1-\mathrm{pF}$ capacitor.

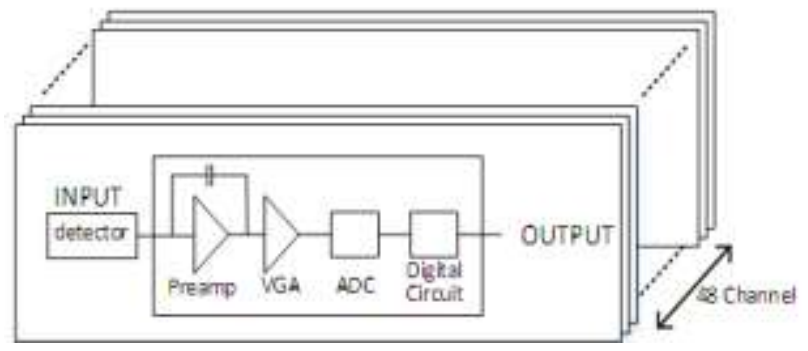

Fig. 1. The 48-channel experimental board.

If the input pulse is with $20 \mathrm{mV}$, the charge that will be deposited on the CSA can be calculated as $\mathrm{Q}_{\text {in }}=\mathrm{CU}=1 \mathrm{pF} \times 20 \mathrm{mV}=20 \mathrm{fC}$. This charge signal will be amplified by the preamplifier in ASIC and then shaped with an ORTEC 570 commercial shaper with various shaping times. The shaping time for this shaping module can be set as $0.5,1,2,3,6$, and $10 \mu \mathrm{s}$. The output of the shaper can be analyzed by the pulse height analysis module, CP 1216/R PHA and CP1216/M Histogram Memory/GP-1B which has the capability of processing 16 signal channels at the same time (Fig. 2).

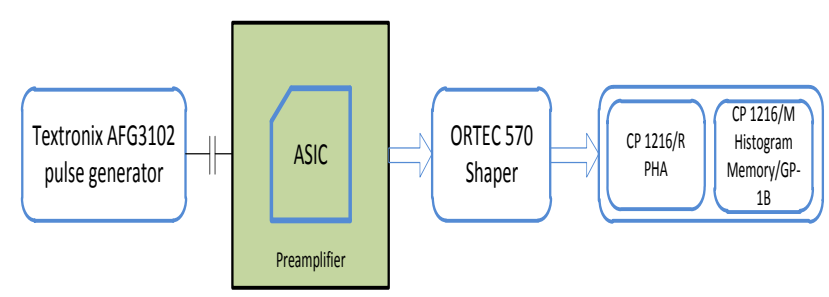

Fig. 2. Setup of ENC measurement of the preamplifier.

\section{RESULTS AND DISCUSSION}

The gamma irradiations were performed with a Co-60 source located at the Heavy Ion Medical Accelerator in Chiba (HIMAC). The Co-60 source emits photons with energies of $3.5574 \times 10^{9} \mathrm{pC}$ and $1.8665 \times 10^{9} \mathrm{pC}$ to the chip in the preamp board. These gamma sources were used to study the ionization effects. For the PET system, the preamp board has 48 input channels and 48 output channels (Fig. 3).

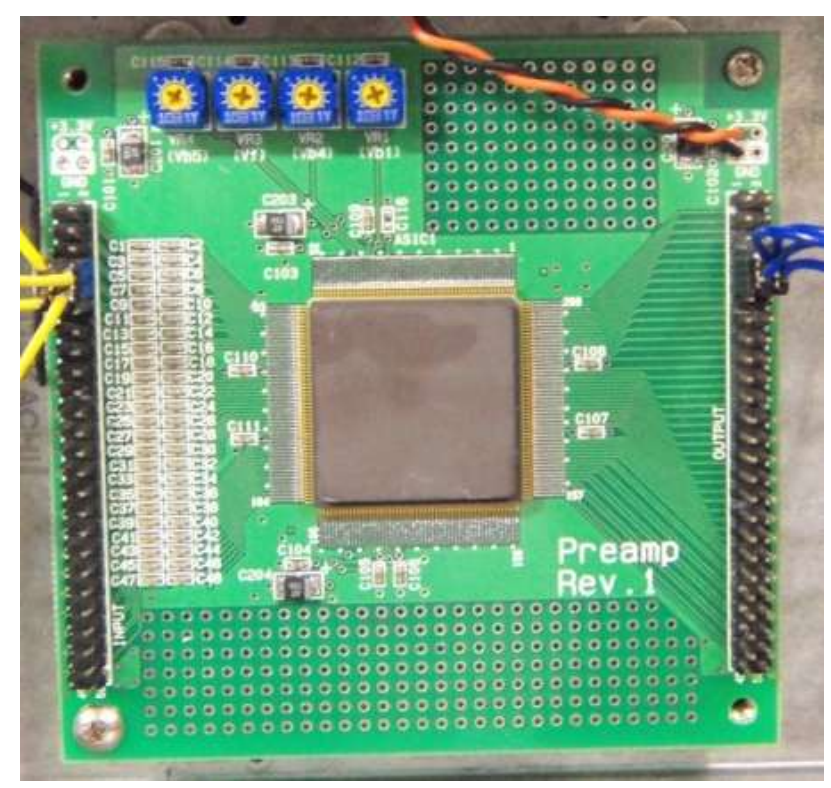

Fig. 3. ASIC Preamp Board.

The output of the shaper was measured with a pulse height analyzer (PHA) with shaping times varied from 0.5 to $10 \mu \mathrm{s}$ while the FWHM was recorded and the position of the peak was determined by the software in the computer. The ENC can be calculated from these two parameters. Figures 4 and 5 show the results of the ENC measurement of the preamplifier before and after irradiation. 


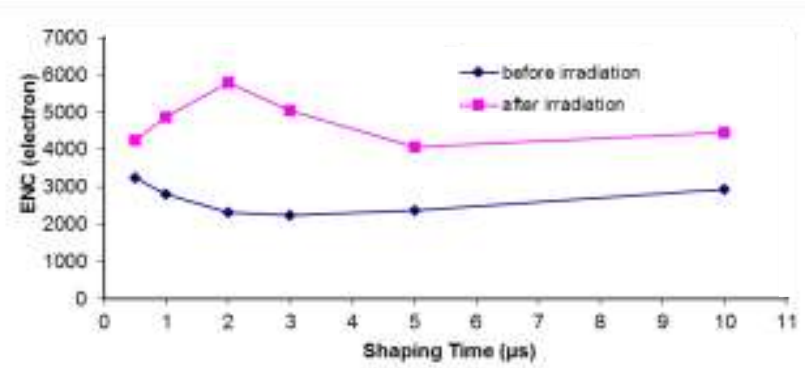

Fig. 4 Result of the ENC measurements of the preamplifier (one channel).

High-energy heavy particles (protons, neutrons, or heavy ions) cause lattice (or displacement) damage by creating vacancies and interstitials. Gamma irradiation causes much less lattice damage. Lattice damage causes degradation of the component parameters [4,5].

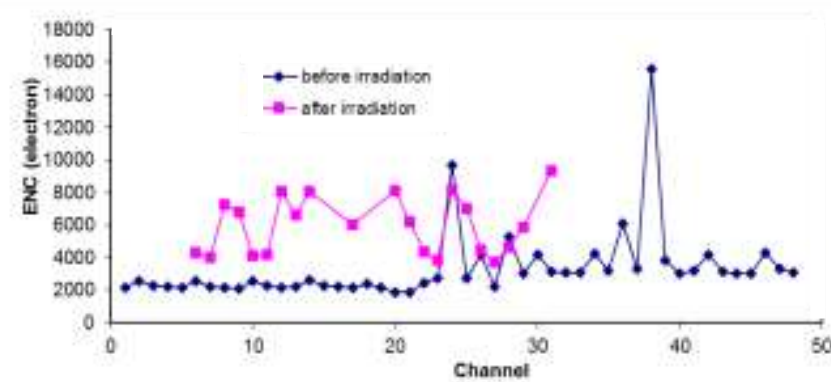

Fig. 5. Result of the ENC measurements of the preamplifier (48 channels).

ENC is a derived quantity - a combined effect of noise current, voltage, and capacitance at the input. The minimum ENC could be obtained at the shaping time where the current and voltage noise contribution are equal [6].

As seen in the ENC characteristic displayed in Fig. 4 and Fig. 5, the minimum ENC is $\sim 2000$ electrons, which was achieved at the shaping time of $0.5 \mu \mathrm{s}$. After applying gamma rays to the preamp board, the ENC measurement results showed some degradation. Most of the channels showed an increased ENC value, and some of those channels were damaged.

Figure 6 illustrates a typical ENC curve which is affected by the noise current, voltage, and capacitance. The minimum ENC is achieved when current noise and voltage noise are same.

By analyzing the ENC curve, it can be concluded that the electronic devices can suffer from long-term radiation effects, and the effects are mostly due to electrons and protons [7]. Furthermore, this cumulative long term ionizing damage due to protons and electrons can cause devices to suffer threshold shifts, increased device leakage (and power consumption), timing changes, and decreased functionality $[8,9]$.

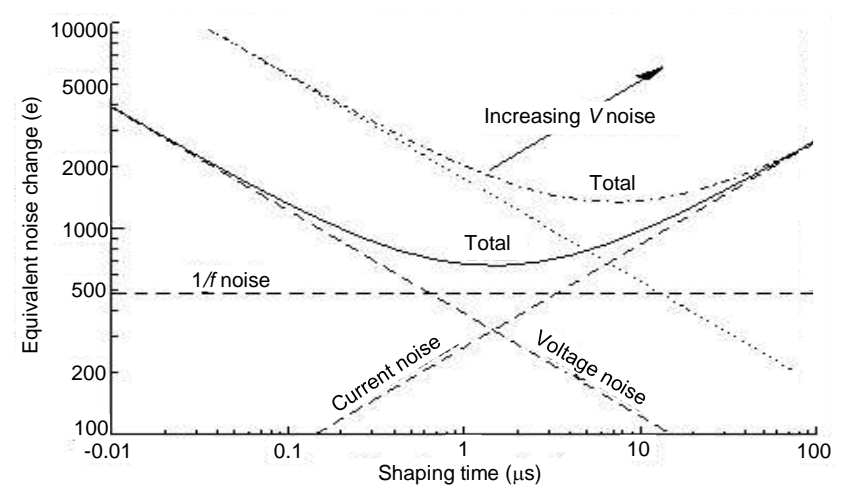

Fig. 6. Typical ENC curve.

It is also important to note that the preamplifier should match the impedance levels between the detector and the subsequent circuit. Therefore, a CSA is needed to convert the charge signal from the detector to a voltage signal that is large enough to be processed by the subsequent electronics due to the low internal gain of the APD in the detector module [10].

There was an attempt to build another preamp board by using discrete components. The preamplifier system was constructed from monolithic ICs [11]. We selected the OPA656 FETinput op-amp from Texas Instrument Corp. with the following features: low input bias current $(2 \mathrm{pA})$, $500 \mathrm{MHz}$ unity-gain bandwidth, low input noise $(7 \mathrm{nV} / \sqrt{\mathrm{Hz}}$, and $1.3 \mathrm{fA} / \sqrt{\mathrm{Hz}}$ ), small size of $2.75 \times 2.6 \times 1.45 \mathrm{~mm}^{3}$, and availability at a low price. The schematic of the preamplifier circuit is shown in Fig. 7.

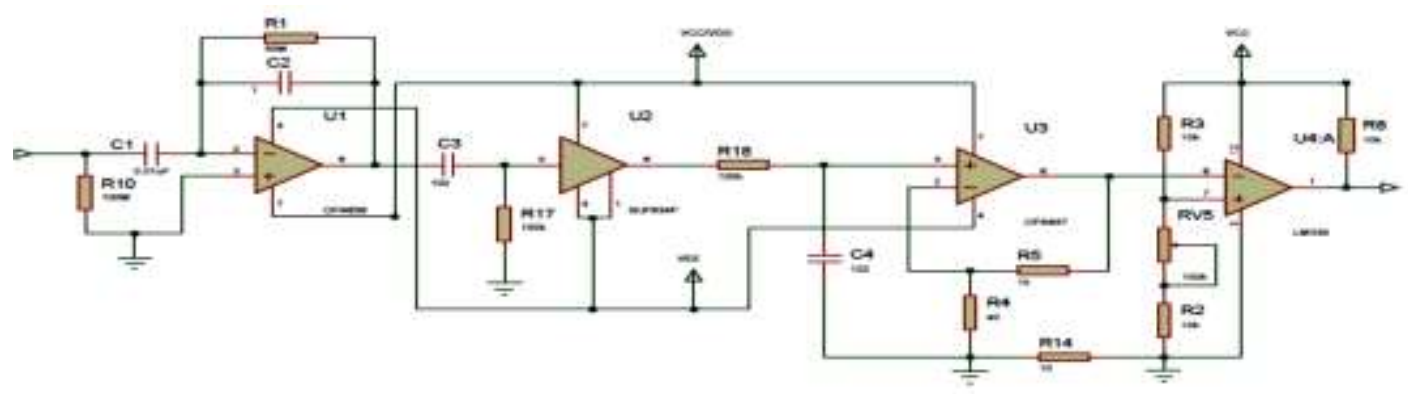

Fig. 7. Schematic of preamplifier circuit. 
The charge-sensitive preamplifiers were operated at $\mathrm{Rf}=500 \mathrm{M} \Omega$ and $\mathrm{Cf}=0.2 \mathrm{pF}$. An amplification stage was included in the preamplifier to adjust the amplitude of the signals corresponding to the input voltage of the ADCs, which span $1 \mathrm{~V}$. The OPA847 op-amp from Texas Instrument Corp. was used for this stage. However, due to the limited time during this research exchange program, only the preamp board systems were constructed from component level, whereas the other parts were integrated with subsystem level.

\section{CONCLUSION}

In this paper, we present the noise performance of front-end electronic systems of a 48-channel front-end ASIC dedicated to gamma-ray detector with LuAG:Pr scintillator. Therefore, the 48-channel time-based ASIC needs to be characterized to validate the possibility of its application in the PET system.

The experimental results and the detailed discussions are also presented. As the first processing unit of front-end systems, chargesensitive preamplifiers (CSA) play an important role in suppressing noise and processing the signal.

Through the experimental results for noise measurements without the detectors, it is indicated that the minimum ENC achieved could be smaller if a shorter shaping time could be applied. With the expected long-duration pulse shape with short and sharp peak pulse shape, shaping amplifiers and other filters are needed to optimize signal to noise and generate more practical pulse shapes for avoiding pileup.

This experiment is not yet completed. Noise measurements still have to be taken for the detectors. Furthermore, in order to make this research more beneficial, continuous development should be expected which include improvements in the performance of CSA, and redesigns of a simple main amplifier, pulse shapers, and PHAs. It is particularly important to develop PHAs using ASIC and FPGA technologies.

\section{ACKNOWLEDGMENT}

The authors would like to thank H. Takahashi, K. Shimazoe, and Y. Nakamura for fruitful discussions and to acknowledge that this work was supported by the Ministry of Education,
Culture, Sports, Science and Technology of Japan (MEXT) and the Nuclear Safety Research Association (NSRA) under the Nuclear Researchers Exchange Program.

\section{REFERENCES}

1. G.F. Knoll, Radiation Detection and Measurements, $3^{\text {rd }}$ ed., Wiley, New York, (2000) 265.

2. K. Shimazoe, T. Orita, Y. Nakamura et al., Nucl. Instrum. Methods Phys. Res. Sect. A 731 (2013) 109.

3. M. Yoshino, J. Kataoka, T. Nakamori et al., Nucl. Instrum. Methods Phys. Res. Sect. A 643 (2011) 57.

4. H. Hillemanns, J.-M. Le Goff, Instrumentation for Applied Physics and Industrial Applications, in: Elementary Particles Detectors for Particles and Radiation. Part 2: Systems and Applications, C.W. Fabjan and H. Schopper (Eds.), Springer-Verlag Berlin Heidelberg, German (2011) 251.

5. A. Camplani, S. Shojaii, H. Shrimali et.al., Scientific Journal Facta Universitatis Series: Electronics and Energetics 27 (2014) 251.

6. A.P. Kashchuk, N.A. Kuchinskii, O.V. Levitskaya et al., Phys. Part. Nucl. Lett. 8 (2011) 40.

7. F. Faccio, Radiation Effects and Hardening by Design in CMOS Technologies, in: Analog Circuit Design: Robust Design, Sigma Delta Converters, RFID, H. Casier, M. Steyaert and A.H.M. van Roermund (eds.), Springer, Netherlands (2011) 69.

8. D. Chen, A. Topper, J.B. Forney et al., Radiat. Eff. Data Work 2012 (2012) 1.

9. V. Bezhenova and A.M. Michalowska-Forsyth, E I Elektrotechnik Und Informationstechnik 133 (2016) 1. http://doi.org/10.1007/s00502015-0380-8.

10. X.C. Fang, C. Hu-Guo, D. Brasse et al., Analog Integr. Circuits Signal Process 66 (2011) 31. http://doi.org/10.1007/978-94-007-0391910.1007/s10470-010-9495-3.

11. M. Nakhostin, Y. Kikuchi, K. Ishii et al., Nucl. Instrum. Methods Phys. Res. Sect. A 614 (2010) 308. 